\section{Hochdosierte stereotaktische Einzeitbestrahlung mit kurativer Intention bei peripherem Bronchialkarzinom ${ }^{1}$}

B. Wahlers ${ }^{1}$, J. Debus ${ }^{2}$, S. Dröge', B. Brendle ${ }^{3}$, L. Freitag ${ }^{4}$

${ }^{1}$ Lungenklinik Hemer, Abt. für Radiologie und Strahlentherapie (Chefarzt: Dr. B. Wahlers)

2 Universität Heidelberg, Radiologische Klinik, Abt. Strahlentherapie (Direktor: Prof. Dr. Dr. M. Wannemacher)

${ }^{3}$ Lungenklinik Hemer, Abt. für Anästhesiologie (Chefarzt: Dr. B. Brendle)

${ }^{4}$ Abt. Pneumologie (Chefarzt: PD Dr. H.-N. Macha)
Zusammenfassung: Beim nichtkleinzelligen Bronchialkarzinom im Stadium T1-T2 ohne Metastasierung erreicht man durch die chirurgische Resektionsbehandlung 5-jahres-Überlebensraten von mindestens $65 \%$. Liegt funktionelle Inoperabilität vor, bietet die Strahlentherapie die zweitbesten Chancen. Bei einem Lungenemphysem mit extrem eingeschränkten Funktionswerten verbietet sich aber auch die konventionelle Strahlentherapie wegen möglicher fibrotischer Reaktionen des Parenchyms. In diesen Fällen bleibt die Möglichkeit einer hochdosierten, stereotaktisch geplanten Einzeitbestrahlung. Bei Verwendung des Studienprotokolls des DKFZ Heidelberg wird in einer einzigen Bestrahlung mit dem Linearbeschleuniger eine Dosis von $24 \mathrm{~Gy}$ im Isozentrum appliziert. Hierbei erhält der sichtbare Tumor eine Dosis von $22 \mathrm{~Gy}$ ( $90 \%$ Isodose umschließend). $20 \mathrm{~Gy}$ werden auf den Tumor mit 6 Millimeter Sicherheitssaum gegeben. Voraussetzung hierfür sind eine aufwändige computergestützte Planung anhand von computertomographischen Querschnittsbildern bei hochexakter Lagerung und Immobilisierung des Patienten. Die Bestrahlung erfolgt idealerweise in Narkose unter Hochfrequenzbeatmung, um Atembewegungen zu vermeiden. Wir berichten mit einer Fallbeschreibung eines Emphysempatienten mit $\mathrm{T}_{2}$-Tumor über diese neue Therapiemöglichkeit.

High Dose, Stereotactic, One-time Radiation Therapy with Curative Intent for Peripheral Lung Cancer: In peripheral non small cell lung cancer without metastasis, surgical resection achieves 5-year survival rates of at least $65 \%$. In functionally inoperable patients radiation therapy offers the second best changes. However, in cases of severe emphysema with severely limited lung function even conventional radiation therapy is prohibited because of possible fibrotic reactions of the lung parenchyma. For such patients high dose rate stereotactic onetime radiation therapy may be an option. According to the study protocol of the DKFZ Heidelberg a dose rate of $24 \mathrm{~Gy}$ at the iso-center is applied with the linear accelerator in a single session. The recognisable tumour is irradiated with $22 \mathrm{~Gy}(90 \%$ isodose included). $20 \mathrm{~Gy}$ are applied to the tumour plus 6 millimeters safety margin. Prerequisites of such a therapy are a detailed computer-based planning using CT scans and an exact positioning with immobilisation of the patient. The irradiation is ideally performed under general anesthesia with high-frequency jetventilation to avoid movements due to breathing. We report on this new therapeutic modality in a patient with lung emphysema having a $\mathrm{T}_{2}$ tumour.

Pneumologie 2000; 54: 486-488

(c) Georg Thieme Verlag Stuttgart · New York

ISSN 0934-8387
Ein heute 59-jähriger Betriebsschlosser kam erstmals 1996 wegen Luftnot bei langjährig bekanntem Lungenemphysem zur stationären Aufnahme. Es lag eine berufliche Asbestexposition vor. Im rechten Spitzenfeld der Lunge fanden sich narbig erscheinende Veränderungen, im linken Lungenoberlappen erschien ein Rundherd tumorverdächtig. Eine Dignitätssicherung gelang damals nicht. Bereits zu diesem Zeitpunkt war der Patient funktionell inoperabel, auf invasivere Diagnostik wurde verzichtet. Verlaufskontrollen zeigten 1998 zunächst keine signifikante Größenzunahme der Herde. Anfang 2000 fanden sich die Indurationsherde in der rechten Lunge unverändert, die Raumforderung im linken Oberlappen hatte aber erheblich an Größe zugenommen. Eine Zelltypsicherung war bronchoskopisch nicht möglich, die Telomeraseaktivität in den Bürstenbiopsien fiel rechts negativ, links deutlich positiv aus. Eine CT-gestützte Punktion des linken Herdes bewies zytologisch ein Plattenepithelkarzinom (Abb.1). Unter der Annahme, dass es sich bei den rechtsseitigen Veränderungen um Narben handelte, ergab sich das Tumorstadium T2, N0, M0. Die Operabilität wurde erneut überprüft. Trotz Ausschöpfung aller medikamentösen Maßnahmen beklagte der Patient jedoch weiterhin Luftnot bei geringster Belastung. Bereits in Ruhe war er wiederholt

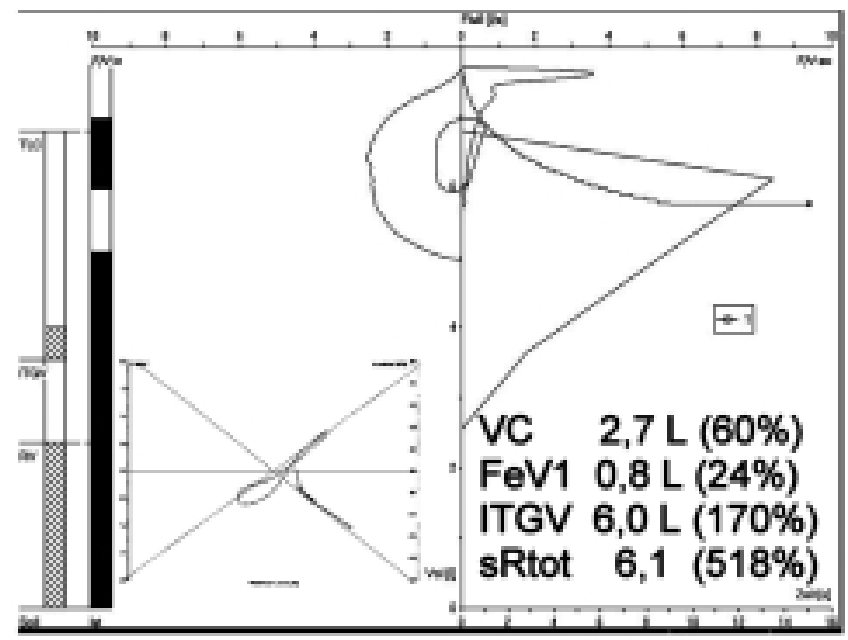

Abb. 1 Emphysemkonstellation mit stark eingeschränkten Funktionswerten.

${ }^{1}$ Herrn PD Dr. med. H.-N. Macha zum 60. Geburtstag gewidmet 


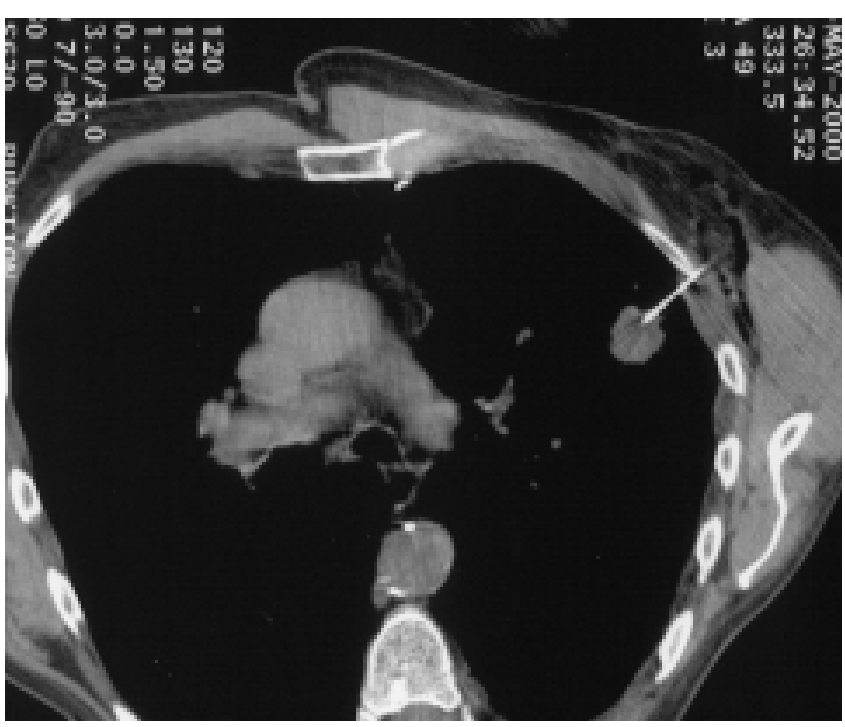

Abb. 2 Zytologische Sicherung des peripheren Bronchialkarzinoms durch CT-gestützte Punktion.

partialinsuffizient. Radiologisch wie funktionsanalytisch fand sich das Vollbild eines Lungenemphysems (Abb. 2). Der Atemstoß betrug $0,8 \mathrm{~L}$ ( $24 \%$ des Solls), es lagen eine irreversible Obstruktion mit einem sRtot von 7,1 kPa*s (600\% der Norm) und eine irreversible Überblähung vor. Der Patient erschien uns auch für einen parenchymsparenden, minimalinvasiven Eingriff nicht operabel. Den letzten Ausschlag zu dieser Entscheidung gab eine schwere respiratorische Insuffizienz durch einen Mantelpneu nach der CT-Punktion. Die Möglichkeit einer konventionell fraktionierten Strahlentherapie wurde wegen des hohen Risikos einer Strahlenpneumonitis oder Fibrose ebenfalls verworfen. Eine stereotaktische Einzeitbestrahlung wurde in der interdisziplinären Tumorkonferenz beschlossen und dem Patienten vorgeschlagen.

Es erfolgte eine computergestützte Planung der Bestrahlung anhand von Querschnittsbildern der CT (Abb.3). Hierbei wurden sechs Marker an der Thoraxwand verwendet. Zur genauen Lagerung des Patienten verwenden wir das computergestützte Positionierungssystem Exa Trac der Firma BrainLab. Über die Markierungspunkte wird die genaue Position des Tumors und des Patienten im Raum errechnet. Die Differenz zwischen Ist- und errechneter Sollposition wird während der Behandlung ständig angezeigt.

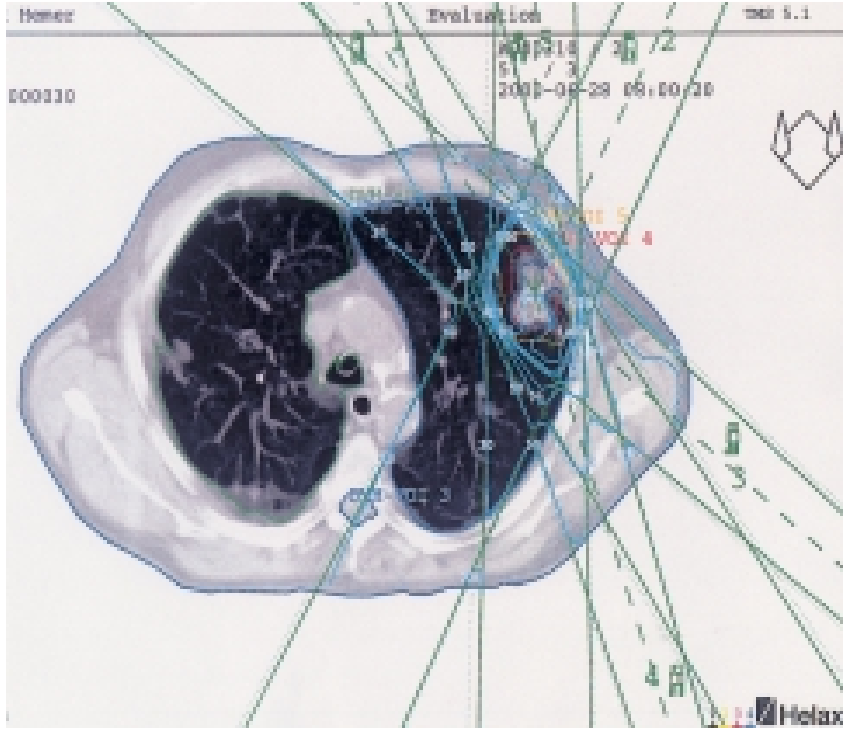

Abb. 3 3-dimensionaler stereotaktischer Bestrahlungsplan.

Die Bestrahlung wurde in intravenöser Allgemeinnarkose durchgeführt. Dabei wurde der Patient mit einem Hochfrequenz-Jet-Ventilator (Acutronic) beatmet, um die Atemexkursionen so gering wie möglich zu halten. Die hochdosierte Einzeitbestrahlung wurde lokal und allgemein subjektiv ohne Nebenwirkung toleriert. Die Kontrolluntersuchung nach sechs Wochen zeigte eine vollständige Remission des Tumors und ganz diskrete, nur fokale Zeichen einer Pneumonitis (Abb. 4au.b).

\section{Diskussion}

Durch chirurgische Behandlung erzielt man bei nicht-kleinzelligen T1-Bronchialkarzinomen 5-Jahres-Überlebensraten um 65\% [1]. Mit kurativ intendierten Strahlentherapieprotokollen kann ein Langzeitüberleben in 40\% der Fälle erreicht werden. Die Strahlentherapie bei Patienten mit schwerem Lungenemphysem ist aber problematisch, da das Risiko einer Strahlenpneumonitis und Fibrose besteht [2]. Mit der stereotaktischen Bestrahlung kann vom theoretischen Ansatz her das Risiko einer größeren Fibrosereaktion minimiert werden. Stereotaktische Verfahren sind bei intrakraniellen Tumoren etabliert, für intrapulmonale Tumoren gibt es bisher wenig Erfahrungen [3,4]. Am Krebsforschungszentrum in Heidelberg wurde ein Protokoll erstellt, um in einer einzigen Sitzung
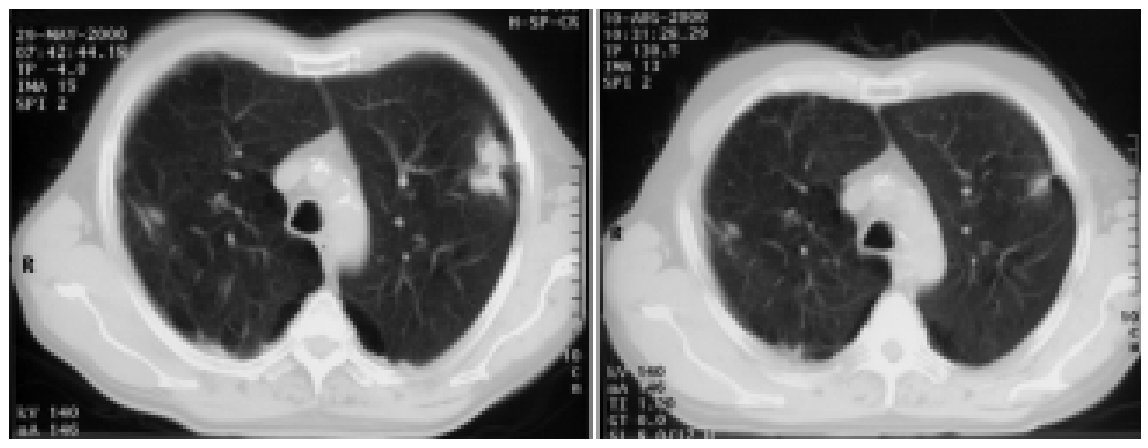

Abb. 4 a Tumor vor Bestrahlung. b Sechs Wochen nach Bestrahlung vollständige Remission des Tumors. Narbige Residuen ohne Fibrose. 
$24 \mathrm{~Gy}$ in die Zielregion zu applizieren [5]. Dem verminderten Langzeitrisiko steht das Problem der schwierigeren Zielplanung gegenüber. Bereits minimale Verschiebungen zwischen der Situation während der Planung und der tatsächlichen Bestrahlung können dazu führen, dass ein Teil des Tumors außerhalb des Bestrahlungsfeldes liegt. Eine exakte Planung und das Anbringen von Referenzmarkern ist unumgänglich. Bei wachen Patienten besteht die Gefahr, dass durch geringe Körperbewegungen das Target verfehlt wird und bei konventioneller Beatmung kommt es atemlageabhängig zu Verschiebungen bis zwei Zentimeter. Um mit einem kleinstmöglichen Zielvolumen arbeiten zu können, führen wir daher die stereotaktische Bestrahlung in Hochfrequenzbeatmung durch. Die Methode ist aufwändig und erfordert eine gute Vorbereitung. Langzeitergebnisse stehen noch aus. Zur Zeit wird eine Phase-I-Studie durchgeführt, um den Stellenwert der stereotaktischen Einzeitbestrahlung zu überprüfen. Sollten sich die vorläufigen Ergebnisse bestätigen, könnte auch Patienten mit T1- bis T2-Tumoren und stark eingeschränkten Lungenfunktionswerten eine potenziell kurative Therapie angeboten werden.

\section{Danksagung}

Die Erweiterung unseres Linearbeschleunigers zur stereotaktischen Bestrahlung wurde mit finanzieller Unterstützung des Landes NRW ermöglicht. Diese Arbeit wird Herrn PD Dr. H.-N. Macha zum 60. Geburtstag gewidmet.

\section{Literatur}

${ }^{1}$ Nesbitt JC, Putnam Jr JB, Walsh GL, Roth JA, Mountain CF. Survival in early-stage non-small cell lung cancer. Ann Thorac Surg 1995; 60: $466-472$

${ }^{2}$ Ono R, Egawa S, Suemasu K, Sakura M, Kitagawa T. Radiotherapy in inoperable stage I lung cancer. Jpn J Clin Oncol 1991; 21: 125

${ }^{3}$ Blomgren H, Lax I, Naslund I, Svanstrom R. Stereotactic high dose fraction radiation therapy of extracranial tumors using an accelerator. Clinical experience of the first thirty-one patients. Acta Oncol 1995; 34: $861-870$

${ }^{4}$ Uematsu M, Shioda A, Tahara K, Fukui T, Yamamoto F, Tsumatori G, Ozeki Y, Aoki T, Watanabe M, Kusano S. Focal, high dose, and fractionated modified stereotactic radiation therapy for lung carcinoma patients: a preliminary experience. Cancer 1998; 82: $1062-1070$

${ }^{5}$ Debus J, Pirzkall A, Schlegel W, Wannenmacher M. Stereotaktische Einzeitbestrahlung (Radiochirurgie). Methoden, Indikationen und Resultate. Strahlenther Onkol 1999; 175: 47-56

\section{Dr. B. Wahlers}

Abteilung für Radiologie und Strahlentherapie Lungenklinik Hemer

Theo-Funccius-Str. 1

58675 Hemer
Förderpreis „Schlafapnoe“ anlässlich der Jahrestagung der DGP in Jena 2001

Die Deutsche Gesellschaft für Pneumologie vergibt auf ihrer Jahrestagung 2001 in Jena einen Förderpreis für wissenschaftliche Arbeiten im Forschungsschwerpunkt Schlafapnoe, der mit DM 12000 dotiert ist. Der Preis dient der Förderung des wissenschaftlichen Nachwuchses in der pneumologischen Schlafmedizin; er wird für die beste wissenschaftliche Arbeit aus diesem Gebiet verliehen. Besonders förderungswürdig sind Arbeiten, die sich mit dem Thema „Tagesschläfrigkeit trotz nCPAPTherapie“ beschäftigen, doch können auch andere Arbeiten zur Diagnostik, Therapie und Ökonomie der Schlafapnoe prämiert werden. Die Bewerber sollten nicht über 40 Jahre alt sein, sie müssen ihre Arbeitsstätte in Deutschland haben oder die Grundlage für die Preisarbeit dort erarbeitet haben. Die eingereichte Arbeit muß in deutscher oder englischer Sprache verfasst sein, und sie darf zu keinem anderen Preis eingereicht sein. Ist die Arbeit bereits publiziert, darf das Erscheinungsdatum der Publikation nicht mehr als ein Jahr vor dem Abgabetermin zur Preiseinreichung zurückliegen. Mehrere thematisch zusammenhängende Publikationen können zusammengefasst und mit einem gemeinsamen Abstrakt versehen eingereicht werden. Der Förderpreis wird von der Selbsthilfegruppe Schlafapnoe/Atemstillstand Essen mit finanzieller Unterstützung durch die Firma ResMed (Mönchengladbach) gestiftet und wird während der Plenarversammlung am 24. März 2001 in Jena verliehen.

Die Bewerber werden gebeten, ihre Arbeit bis zum 15. 1. 2001 in 4facher Ausfertigung dem Geschäftsführer der Gesellschaft, Herrn Prof. Adrian Gillissen, Robert-KochKlinik, Nikolai-Rumjanzew-Straße 100, 04207 Leipzig, zu schicken. 